\title{
Urethral opening pressure in patients with myelodysplasia
}

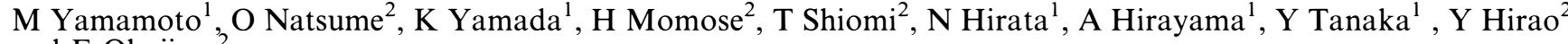 \\ and E Okajima ${ }^{2}$ \\ ${ }^{1}$ Department of Urology, Hoshigaoka Koseinenkin Hospital, 4-8-1, Hoshigaoka, Hirakata, Osaka, 573; ${ }^{2}$ Department \\ of Urology, Nara Medical University, 840, Shijo-cho, Kashihara, Nara, 634, Japan
}

\begin{abstract}
We evaluated 60 patients with myelodysplasia using the urethral opening pressure (UOP) which is defined as the total intravesical pressure at the moment when urine passes through the external sphincter by the Credé or the Valsalva maneuver. Twenty nine of the patients showed UOP value greater than $35 \mathrm{cmH}_{2} \mathrm{O}$ and 31 below $35 \mathrm{cmH}_{2} \mathrm{O}$. In the high pressure group, patients showed a tendency to preserve sphincter activity and had mild paralysis of the lower extremities. In contrast, most of patients in the low pressure group showed an underactive sphincter and severe paralysis of the lower extremities. The incidence of deterioration of the upper urinary tract, vesicoureteral reflux (VUR) and bladder deformity were significantly high in the high pressure group than those in the low pressure group $(75.9 \%$ vs $16.1 \%, 72.4 \%$ vs $22.6 \%, 82.8 \%$ vs $29.0 \%$, respectively, $P<0.05$ ). During the follow-up period (42 months on average), the voiding methods were changed in 15 patients from the Credé maneuver to clean intermittent catheterization, and total of 12 operations were performed (10 antiVUR operation and two with an ileocystoplasty). Finally, approximately $90 \%$ of the patients in both the high and the low pressure groups were evaluated as improved or stable in the upper urinary tract, VUR and bladder deformity after conservative or surgical managements. UOP is considered to be one of the useful parameters to select the high risk patient in the urological management of myelodysplastic patients.
\end{abstract}

Keywords: urethral opening pressure; myelodysplasia; urinary tract deterioration

\section{Introduction}

The most important issue in the urological management of myelodysplastic patients is to prevent deterioration of their renal function. For many years, it has been emphasized that a high intravesical pressure is a significant cause of morbidity in myelodysplastic patients. ${ }^{1-3}$ They can develop urological complications such as upper urinary tract deterioration, vesicoureteral reflux (VUR) and bladder deformity.

So far, we have treated more than 300 myelodysplastic patients. ${ }^{4}$ In some of them the upper urinary tract condition worsened even if there appeared to be a similar degree of paralysis of bladder function and lower extremities to other patients. Some studies have been demonstrated to diagnose patients at high risk for upper urinary tract deterioration. ${ }^{5-11}$

In this report, we describe a new and practical urodynamic assessment, urethral opening pressure (UOP), which is defined as the total intrevesical pressure at the moment when urine passes through the external sphincter by the Credé or the Valsalva maneuver, for myelodysplastic patients.

Correspondence: M Yamamoto

\section{Materials and methods}

We investigated 60 patients with myelodysplasia who have been followed up at our institutes. Of these patients 29 were males and 31 females. Mean patient age at the initial evaluation was 14 years (range 3 to 38 years) and follow-up period ranged from 36 to 48 months (mean 42 months). All patients had an underactive detrusor.

External sphincter activity was classified into three categories; (1) active moderate, (2) active weak, and (3) underactive. ${ }^{4}$ Seven patients had an active moderate sphincter, 29 an active weak sphincter, and 24 an underactive sphincter. The degree of paralysis of the lower extremities was expressed according to Sharrard's classification. ${ }^{12}$

Sharrard group is defined, I: paralysis below the twelfth thoracic root; II: paralysis below the first or second lumbar root; III: paralysis below the third or fourth lumbar root; IV: paralysis below the fifth lumbar root; V: paralysis below the first sacral root; VI: no lower limb paralysis.

UOP measurement was performed as previously described (Figure 1). ${ }^{5}$ In brief, the patient is set in the supine position and two catheters are inserted transurethrally, one is an $8 \mathrm{~F}$ single lumen catheter for filling up the bladder and the other is an 18 gauge 
A

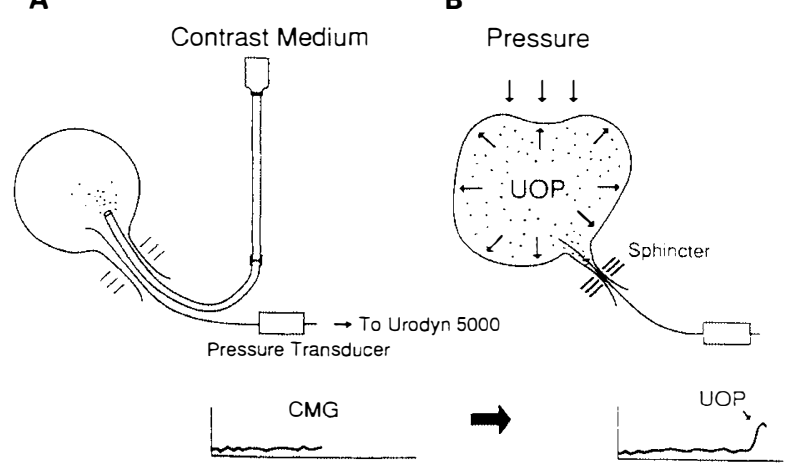

Figure 1 Schema of measurement of urethral opening pressure (UOP). (A) Two catheters are inserted into the bladder transurethrally. An $8 \mathrm{~F}$ catheter is for instillation of contrast medium. Cystometrogram $(\mathrm{CMG})$ is recorded via an $18 \mathrm{G}$ epidural tube. (B) After removal of the catheter for filling, the patient attempts to void by the Crede or the Valsalva maneuver. Urethral opening pressure is defined as the total vesical pressure when urine passes through the external sphincter

Table 1 Characteristics of 60 patients with myelodysplasia

\begin{tabular}{lcr}
\hline No. of Pts. & 60 & \multicolumn{2}{c}{$(\mathrm{M}: \mathrm{F}=29: 31)$} \\
Age (years) & $14.5 \pm 8.9$ & $(3-38)$ \\
Follow up (mos.) & $42.2 \pm 3.0$ & $(36-48)$ \\
Sphincter activity & 24 \\
$\quad$ Underactive & 29 \\
Active weak & 24 \\
Active moderate & 7 \\
Sharrard's classification & \\
Group I & 3 \\
Group II & 3 \\
Group III & 8 \\
Group IV & 18 \\
Group V & 16 \\
Group VI & 12 \\
\hline
\end{tabular}

epidural tube for monitoring the intravesical pressure. Under the fluoroscope, contrast medium is infused into the bladder at a rate of $20 \mathrm{ml}$ per min. When the patient complains of lower abdominal discomfort due to the full bladder, the catheter for filling is removed. Then patient attempts to void by the Crede or the Valsalva maneuver and the intravesical pressure is recorded at the moment when urine (contrast medium) passes through the external sphincter. This pressure is defined as UOP. We have confirmed that the value of UOP is not influenced by the infused volume and is reproductible at any examination. ${ }^{5}$

All patients underwent excretory urography and voiding cystourethrography on the same day of UOP measurement (initial examination) with periodic reevaluations $(1-2 /$ year $)$.
We divided the patients into two groups based on UOP value (Vide infra), and analyzed these two groups on the frequency of deterioration of the upper urinary tract, VUR and bladder deformity.

Patients with (1) lower ureteral ectasis to hydronephrosis, (2) VUR greater than grade I according to the international classification, and (3) bladder deformity greater than grade I according to Ogawa's classification $^{13}$ was evaluated as abnormal. We also compared these three parameters in the initial and final examinations. Chi-square test was used for statistical analysis.

\section{Results}

The distributions of UOP in relation to upper urinary tract, VUR and bladder deformity are shown in Figure 2. Normal cases are indicated by open circles and cases with abnormal findings by closed circles. The border line of UOP between normal and abnormal cases appeared around 35 to $40 \mathrm{cmH}_{2} \mathrm{O}$; we estimated accuracy of UOP at every value. The highest accuracy was obtained at UOP value $35 \mathrm{cmH}_{2} \mathrm{O}$ for bladder deformity $(76.7 \%)$ and UOP value $37 \mathrm{cmH}_{2} \mathrm{O}$ for upper urinary tract deterioration and VUR $(80.0 \%$ and $76.7 \%$, respectively). Then we divided the patients into two groups by UOP value $35 \mathrm{cmH}_{2} \mathrm{O}$. Thirty one

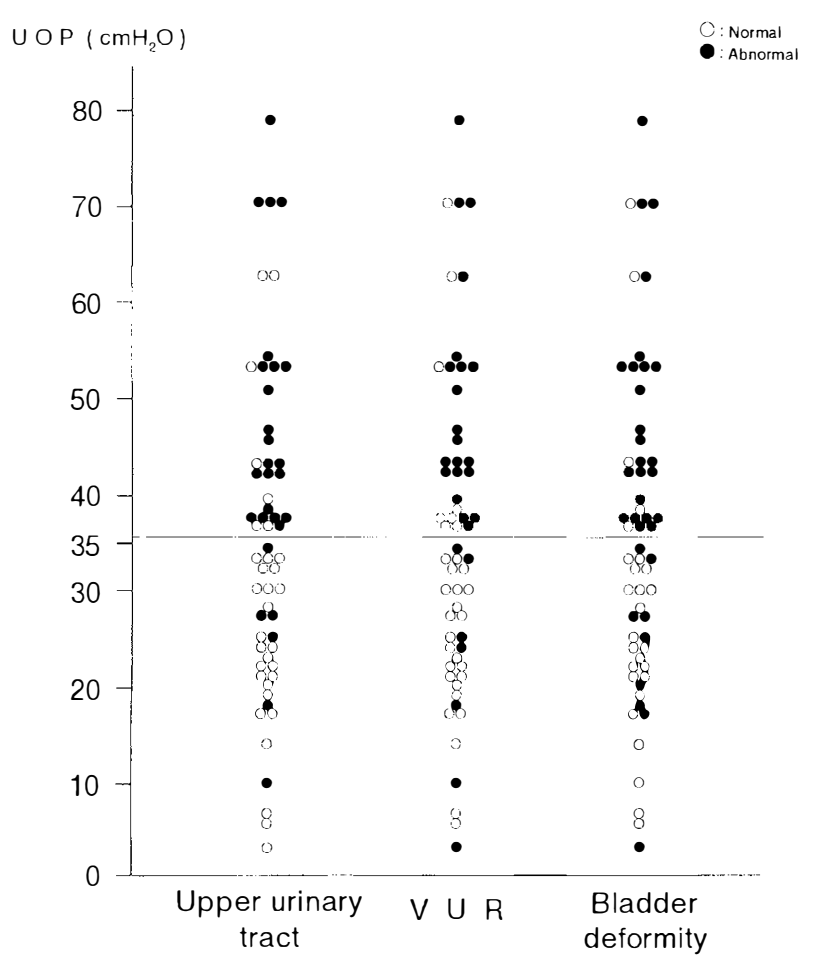

Figure 2 Distributions of UOP values of 60 myelodysplastic patients in relation to the upper urinary tract, vesicoureteral reflux (VUR) and bladder deformity. Open circle indicates normal cases and closed circle indicates those with abnormal findings. UOP: urethral opening pressure 
patients with UOP values below $35 \mathrm{cmH}_{2} \mathrm{O}$ were designated as the low pressure group and 29 with UOP values greater than $35 \mathrm{cmH}_{2} \mathrm{O}$ as the high pressure group. UOP values of the low pressure group

\begin{tabular}{|c|c|c|c|c|}
\hline \multirow{2}{*}{$\begin{array}{c}\text { Sharrard's } \\
\text { classification }\end{array}$} & \multicolumn{3}{|c|}{ External Sphincter activity } & \multirow{2}{*}{ Total } \\
\hline & Underactive & $\begin{array}{r}\text { Active } \\
\text { weak }\end{array}$ & $\begin{array}{c}\text { Active } \\
\text { moderate }\end{array}$ & \\
\hline Group I & m & & & 3 \\
\hline Group II & e & 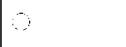 & & 3 \\
\hline Group II & 6 & $\infty$ & - & 8 \\
\hline Group $\mathrm{N}$ & 00 & 00000 & & 18 \\
\hline Group $\mathrm{V}$ & $\begin{array}{l}00 \\
00\end{array}$ & 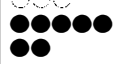 & - & 16 \\
\hline Group VI & & 00000 & $\bullet \bullet \bullet \bullet$ & 12 \\
\hline Total & 24 & 29 & 7 & 60 \\
\hline
\end{tabular}

O: $\cup O P<35 \mathrm{cmH}_{2} \mathrm{O}(\mathrm{n}=31)$

- $\cup O P \geqq 35 \mathrm{cmH}_{2} \mathrm{O}(\mathrm{n}=29)$

Figure 3 Distribution of patients according to sphincter activity and degree of paralysis of lower extremities of 60 patients with myelodysplasia. Note that patients with active sphincter and mild paralysis were frequent in the high pressure group ranged from 4 to $34 \mathrm{cmH}_{2} \mathrm{O}$ (mean $\pm \mathrm{SD}$; $22.8 \pm 8.3 \mathrm{cmH}_{2} \mathrm{O}$ ) and 37 to $79 \mathrm{cmH}_{2} \mathrm{O}$ (mean $\pm \mathrm{SD}$; $\left.49.2 \pm 11.7 \mathrm{cmH}_{2} \mathrm{O}\right)$. The distribution of patients classified according to sphincter activity and degree of paralysis of the lower extremities is striking (Figure 3). The patients who had mild paralysis of lower extremities (Sharrard groups V and VI) showed a tendency to preserve sphincter activity. The majority of these patients belonged to the high pressure group (closed circles). In contrast, most of patients with severe paralysis of the lower extremities had an underactive sphincter. Patients belonging to the low pressure group (open circles) were frequent.

The incidence of abnormal upper urinary tract, VUR and bladder deformity are shown in Table 2. Twenty two patients $(75.9 \%)$ in the high pressure group had an abnormal urinary tract compared to only $5(16.1 \%)$ in the lower pressure group. VUR was observed in 21 patients $(72.4 \%)$ in the high pressure group and only $7(22.6 \%)$ in the low pressure group. Bladder deformity was demonstrated in 24 patients $(82.8 \%)$ in the high pressure group and $9(29.0 \%)$ in the low pressure group. All the incidences in the high pressure group were significantly higher than those in the low pressure group $(P<0.05)$.

During the follow-up period, voiding methods were changed in 15 patients (nine in the low pressure group and six in the high pressure group) from the Credé maneuver to clean intermittent catheterization (CIC) (Table 3). Eight of these 15 patients showed a progression of changes in the upper urinary tract and/or VUR and the remaining seven who had a normal upper urinary tract had their voiding methods changed for their convenience. Finally, all of the patients in the high pressure group were managed with

Table 2 Incidence of an abnormal upper urinary tract, VUR and bladder deformity in 60 myelodysplastic patients at the initial examination

\begin{tabular}{|c|c|c|c|}
\hline \multirow[b]{2}{*}{ Clinical findings } & \multicolumn{3}{|c|}{ No. of abnormal findings $(\%)$} \\
\hline & $\begin{array}{c}U O P<35 \mathrm{CmH}_{2} \mathrm{O} \\
(n=31)\end{array}$ & $\begin{array}{c}U O P \geqslant 35 \mathrm{cmH}_{2} \mathrm{O} \\
(n=29)\end{array}$ & $\begin{array}{c}\text { Total } \\
(n=60)\end{array}$ \\
\hline Upper urinary tract & $5 \quad(16.1)$ & $22 \quad(75.9)^{*}$ & $27 \quad(45.0)$ \\
\hline Vesicoureteral reflux & $7 \quad(22.6)$ & $21 \quad(72.4)^{*}$ & $28 \quad(46.7)$ \\
\hline Bladder deformity & $9 \quad(29.0)$ & $24 \quad(82.8)^{*}$ & $33 \quad(55.0)$ \\
\hline
\end{tabular}

*: $P<0.01$ (Chi-square test)

Table 3 Alteration of voiding method in 60 patients with myelodysplasia

\begin{tabular}{|c|c|c|c|c|c|}
\hline \multicolumn{3}{|c|}{ Voiding method at initial examination } & \multicolumn{3}{|c|}{ Voiding method at final examination } \\
\hline \multirow[b]{2}{*}{$U O P$} & \multicolumn{2}{|c|}{ Voiding method (No. Pts.) } & \multirow[b]{2}{*}{$U O P$} & \multicolumn{2}{|c|}{ Voiding method (No. Pts.) } \\
\hline & $C I C$ & Credé or Valsalva & & $C I C$ & Crede or Valsalva \\
\hline $\mathrm{UOP}<35 \mathrm{cmH}_{2} \mathrm{O}$ & 10 & 21 & $\mathrm{UOP}<35 \mathrm{cmH}_{2} \mathrm{O}$ & 19 & 12 \\
\hline $\mathrm{UOP} \geqslant 35 \mathrm{cmH}_{2} \mathrm{O}$ & 23 & 6 & $\mathrm{UOP} \geqslant 35 \mathrm{cmH}_{2} \mathrm{O}$ & 29 & 0 \\
\hline Total & 33 & 27 & Total & 48 & 12 \\
\hline
\end{tabular}


Table 4 Operations performed on follow up period

\begin{tabular}{lcc}
\hline & \multicolumn{2}{c}{ Operations $($ No. Pts) } \\
\cline { 2 - 3 }$U$ Anti $\frac{\text { VUR }}{2}$ & 3 & Ileocystoplasty \\
\hline $\mathrm{UOP}<35 \mathrm{cmH}_{2} \mathrm{O}$ & 7 & 0 \\
$\mathrm{UOP} \geqslant 35 \mathrm{~cm} \mathrm{H}_{2} \mathrm{O}$ & 10 & 2 \\
Total & & 2 \\
\hline
\end{tabular}

CIC. On the other hand, 12 patients $(38.7 \%)$ in the low pressure group were still employing the Crede maneuver. Fifteen patients (low pressure group 6, high pressure group 9) combined CIC with anticholinergic drug therapy.

A total of 12 operations was performed in the follow-up period. Ten patients (three in the low pressure group and seven in the high pressure group) underwent an antiVUR operation. Ileocystoplasty was performed for two patients in the high pressure group (Table 4).

In the low pressure group, three patients showed improvement in the upper urinary tract changes. Only one who developed mild lower ureteral ectasis was classified as worse. VUR disappeared in two patients and newly developed in three patients (but all three patients had grade I reflux). Three patients showed progression of bladder deformity (Table 5A). In the high pressure group, hydronephrosis improved in nine patients and progressed in two patients. VUR disappeared in 11 patients. Bladder deformity became less marked in two patients and worse in three patients (Table 5B). Finally, approximately $90 \%$ of the patients were evaluated regarding improvement or stability of the upper urinary tract, VUR and bladder deformity after conservative and surgical management.

\section{Discussion}

Myelodysplasia complicates various problems in the field of neurosurgery, orthopedics and urology. Urologically, vesicourethral dysfunction is a serious problem in the management of myelodysplastic patients. Patients with vesicourethral dysfunction are at risk of developing upper urinary tract deterioration, VUR and bladder deformity. Smith ${ }^{14}$ reported that $58 \%$ of untreated patients with myelodysplasia developed upper urinary tract deterioration by the age of 3 years. Some studies have been reported to detect patients at high risk for upper urinary tract deterioration..$^{5-11}$ McGuire et al $^{7}$ demonstrated that the bladder pressure at which urethral leakage occurred was a useful parameter to predict the high risk patient with myelodysplasia. They reported that $81 \%$ of the patients who had a leak pressure greater than $40 \mathrm{cmH}_{2} \mathrm{O}$ showed upper urinary tract deterioration, whereas this occurred in $10 \%$ of the patients with leak pressure below $40 \mathrm{cmH}_{2} \mathrm{O}$. Kobayashi et al ${ }^{10}$ reviewed 91 patients with myelodysplasia and showed that a maximum urethral closing pressure of greater than $50 \mathrm{cmH}_{2} \mathrm{O}$ was a significant factor that affects the incidence of VUR. Churchill et al ${ }^{15}$ reported that patients whose urethral closing pressure was below $35 \mathrm{cmH}_{2} \mathrm{O}$ had little risk to develop even bladder trabeculation. All of these studies are thought to be of static examinations. On the other hand, Bauer et $a l^{8}$ examined 36 infants with myelodysplasia and showed that $72 \%$ of patients with dyssynergia of the detrusorexternal sphincter had or were later found to have hydroureteronephrosis; this was seen in $22 \%$ with synergy and $11 \%$ with absent external sphincter activity. They concluded that detrusor sphincter dyssynergia is a risk factor for the deterioration of the upper urinary tract. However, they did not express the degree of dyssynergia as a numerical value.

In this report, we defined the urethral opening pressure (UOP) as total intravesical pressure at the moment when urine passes through the external sphincter by the Crede or the Valsalva maneuver. We consider that this method gives the information of the dynamic phase of urination that patients have performed at the ordinary time and we can express the degree of total vesical resistance as a numerical value. Decter $e t \mathrm{al}^{16}$ reported that the clinical management of the measurement of the leak point pressure during water cystometry is influenced by the catheter size, and it depends upon the technique and the rate of infusion.

Table 5 Final evaluations on upper urinary tract, VUR and bladder deformity in 60 patients with myelodysplasia

\begin{tabular}{|c|c|c|c|}
\hline \multirow[b]{2}{*}{ Clinical findings } & \multicolumn{3}{|c|}{ Clinical evaluation (No Pts. (\%)) } \\
\hline & Improved & Stable & Worse \\
\hline \multicolumn{4}{|c|}{ (A) $\mathrm{UOP}<35 \mathrm{cmH}_{2} \mathrm{O}(\mathrm{n}=31)$} \\
\hline Upper urinary tract & $3 \quad(9.7)$ & $(87.1)$ & $(3.2)$ \\
\hline Vesicoureteral reflux & $2 \quad(6.4)$ & $(72.4)$ & $(9.7)$ \\
\hline Bladder deformity & $1 \quad(3.2)$ & $24 \quad(82.8)$ & $(9.7)$ \\
\hline \multicolumn{4}{|c|}{ (B) $U O P \geqslant 35 \mathrm{cmH}_{2} \mathrm{O}(\mathrm{n}=29)$} \\
\hline Upper urinary tract & $(31.0)$ & $(62.1)$ & (6.9) \\
\hline Vesicoureteral reflux & $11 \quad(37.9)$ & $(51.7)$ & $(10.4)$ \\
\hline Bladder deformity & $(6.9)$ & $(82.7)$ & $(10.4)$ \\
\hline
\end{tabular}


In our method of measurement of UOP, instillation of contrast medium is stopped and only an 18 gauge epidural tube remains for the determination of UOP value, so we can reduce the technical influence to the minimum.

We analyzed 60 myelodysplastic patients by dividing them into two groups at UOP value $35 \mathrm{cmH}_{2} \mathrm{O}$, and revealed the incidence of upper urinary tract deterioration, VUR and bladder deformity were significantly high in the patients with a UOP value greater than $35 \mathrm{cmH}_{2} \mathrm{O}$ than those of a UOP value below $35 \mathrm{cmH}_{2} \mathrm{O} \quad(P<0.01)$. We also demonstrated that the patients who had a UOP value greater than $35 \mathrm{cmH}_{2} \mathrm{O}$ showed a tendency to retain external sphincter activity and suffered mild paralysis of the lower extremities. There have been a few reports referring to the relation between the degree of paresis of the lower extremities and bladder dysfunction in myelodysplastic patients. ${ }^{4,17}$ Our results suggested that patients with (1) an UOP value of greater than $35 \mathrm{cmH}_{2} \mathrm{O}$, (2) remaining sphincter activity and (3) mild paralysis of the lower extremities, have a great risk for upper urinary tract deterioration.

We followed up these 60 patients for 42 months on average. Fifteen patients changed their voiding method from the Crede or the Valsalva maneuver to CIC, and 12 patients underwent surgical treatment (10 antiVUR operation and two ileocystoplasty) during the follow-up period. At the final evaluations, approximately $90 \%$ of patients evaluated had improved or were stable regarding the upper urinary tract, VUR and bladder deformity. These results indicate that the UOP value is a good guide for the management to prevent progression of upper urinary tract deterioration in myelodysplastic patients.

We do not discuss the correlation between UOP and other parameters, such as leak point pressure ${ }^{7,9}$ and maximum urethral closing pressure. ${ }^{0,15}$ However, UOP reflects the degree of total vesical outlet resistance in the dynamic phase of urination, hence the UOP is considered to be a useful parameter to select the high risk patient in the management of myelodysplastic patients. To confirm the predictive value of UOP measurement, a further prospective study will be demanded.

\section{References}

1 McGuire EJ, Woodside JR, Borden TA. Upper urinary tract deterioration in patients with myelodysplasia and detrusor hypertonia: a followup study. J Urol 1988; 129: 823-826.

2 Sidi AA, Dykstra DD, Gonzalez R. The value of urodynamic testing in the management of neonates with myelodysplasia: A prospective study. J Urol 1986; 135: 90-93.

3 Geraniotis E, Koff SA, Enrile B. The prophylactic use of clean intermittent catheterization in the treatment of infant and young children with myelomeningocele and neurogenic bladder dysfunction. J Urol 1988; 139: 85-86.

4 Yamada K et al. Urological management of spina bifida. Spine Spinal Cord 1988; 1: 665-669.

5 Natsume $\mathrm{O}$ et al. Urethral opening pressure as a parameter for introduction of proper voiding modality in myelodysplastic patients. Jpn J Urol 1994; 85:1484-1493.

6 Galloway NTM, Mekras JA, Helms M, Webster GD. An objective score to predict upper urinary tract deterioration in myelodysplasia. J Urol 1981; 145: 535-537.

7 McGuire EJ, Woodside JR, Borden TA, Weiss RM. Prognostic value of urodynamic testing in myelodysplastic patients. $J$ Urol 1981; 126: $205-209$.

8 Bauer SB et al. Predictive value of urodynamic evaluation in newborns with myelodysplasia. JAMA 1984; 252: 650-652.

9 Ghoniem GM, Roach MB, Lewis VH, Harmon EP. The value of leak pressure and bladder compliance in the urodynamic evaluation of meningomyelocele patients. J Urol 1992; 144: $1440-1442$.

10 Kobayashi S et al. Relevance of detrusor hyperreflexia, vesical compliance and urethral pressure to the occurrence of vesicoureteral reflux in myelodysplastic patients. J Urol 1992; 147: $413-$ 415.

11 Perez LM, Khoury J, Webster GD. The value of urodynamic studies in infants less than 1 year old with congenital spinal dysraphism. J Urol 1992; 148: 584-587.

12 Sharrard WJW. Posterior iliopsoas translocation in the treatment of paralytic disloction of the hip. J Bone Joint Sur 1964; 45-B: $426-444$.

13 Ogawa T. Bladder deformities in patients with neurogenic bladder dysfunction. Urol Int 1994; 7 (suppl.1.): 59-62.

14 Smith ED. Urinary prognosis in spina bifida. J Urol 1987; 108 $815-817$.

15 Churchill BM, Gilmour RF, Williot P. Urodynamics. Ped Clin N Amer 1987; 34: 1133 - 1157 .

16 Decter DP, Harpster L. Pitfalls in determination of leak point pressure. J Urol 1992; 148: 588-591.

17 Dator DP et al. Urodynamic dysfunction in walking myelodysplastic children. J Urol 1992; 148: $362-365$. 